\title{
Iterative LQG Controller Design Through Closed-Loop Identification
}

\section{Min-Hung Hsiao, ${ }^{1}$ Jen-Kuang Huang, ${ }^{2}$ and David E. Cox ${ }^{3}$}

This paper presents an iterative $L Q G$ controller design approach for a linear stochastic system with an uncertain openloop model and unknown noise statistics. This approach consists of closed-loop identification and controller redesign cycles. In each cycle, the closed-loop identification method is used to

\footnotetext{
' Associate Professor. Department of Mechanical Engineering, Taipei Institute of Technology, Taiwan.

${ }_{2}$ Associate Professor, Dept. of Mechanical Engineering, Old Dominion University. Norfolk. VA 23529-0247. Assoc. Mern. ASME.

${ }^{3}$ Research Engineer, Guidance and Control Branch. Flight Dynamics and Control Division, NASA Langley Research Center, Hampton, VA 23681-0001.

Contributed by the Dynamic Systems and Control Division of THE AMERICAN SOCIETY OF MECHANICAL ENGINEERs. Manuscript received by the DSCD April 2. 1994. Associate Technical Editor: A. Ray.
} 
identify an open-loop model and a steady-state Kalman filter gain from closed-loop input/output test data obtained by using a feedback LQG controller designed from the previous cycle. Then the identified open-loop model is used to redesign the state feedback. The state feedback and the identified Kalman filter gain are used to form an updated $L Q G$ controller for the next cycle. This iterative process continues until the updated controller converges. The proposed controller design is demonstrated by numerical simulations and experiments on a highly unstable large-gap magnetic suspension system.

\section{Introduction}

Classical Linear Quadratic Gaussian (LQG) controllers are designed by solving two separate, but dual problems: the Linear Quadratic Regulator (LQR) design and Kalman filter (i.e., optimal state estimator) design. The performance of the controllers relies on an accurate open-loop model for the $L Q R$ and an accurate estimate of the measurement and process noise statistics for the Kalman filter. It is difficult to obtain an accurate model through analysis for some systems, and an accurate estimate of the noise statistics through testing for most systems. Furthermore, the noise statistics may be related to the controller if part of the measurement and process noise are generated by the sensor and actuator amplifiers, respectively. To overcome these problems, we present an iterative LQG controller design approach for a linear stochastic system with an uncertain openloop model and unknown noise statistics. This approach consists of closed-loop identification and controller redesign cycles. The closed-loop identification method can simultaneously identify the open-loop model and the Kalman filter gain under the closed-loop operation with a known dynamic controller. Then the identified open-loop model is used for the $L Q R$ design. The LQR and the identified Kalman filter gain are used to form the updated LQG controller for the next closed-loop identification. The process continues until the updated LQG controller converges.

For system identification, several methods (Chen et al., 1992a, 1992b, 1993; Phan et al., 1991; Juang et al., 1993) have been introduced recently to identify the state-space model of a linear system and the Kalman filter. Typically the system is under open-loop excitation with an uncorrelated white noise input. For an unstable system, the input/output data are not available while it is under an open-loop operation. To directly use these methods, we have to design a controller and an input signal for the closed-loop system so that the input signal to the open-loop system is almost white. Unfortunately, this is very difficult. On the other hand, some identification methods (Phan et al., 1992; Liu and Skelton, 1990) have been proposed recently for identifying a system under closed-loop operation. However, they have several shortcomings. First, the Kalman filter gain cannot be simultaneously identified because they are applied only for deterministic systems. In Phan et al. (1992), no recursive form was derived for computing the open-loop system Markov parameters, and in Liu and Skelton (1990), the approach is based on system pulse response. In this paper, a recursive form for computing the open-loop system and Kalman filter Markov parameters is derived for stochastic systems with random excitation.

For a system under closed-loop operation, a novel approach for identifying the open-loop model and Kalman filter gain is presented. First, we derive the relation between closed-loop state-space and AutoRegressive with eXogeneous (ARX) models for stochastic systems. From the derivation, it can be seen that a state-space model can be represented by an ARX model if the order of the ARX model is chosen large enough. Since the relation between the input/output data and the system parameters of an ARX model is linear, a linear programming approach like least-square methods, can be used for the ARX model parameter estimation. Second, we derive the algorithm to compute the open-loop system and Kalman filter Markov parameters from the estimated ARX model parameters. In this step, we first compute the closed-loop system and Kalman filter Markov parameters from the estimated ARX model parameters. Then the open-loop system and Kalman filter Markov parameters are computed from the closed-loop system and Kalman filter Markov parameters and the known controller Markov parameters. Third, the state-space model for the open-loop system is realized from the open-loop Markov parameters through the singular value decomposition method (Chen et al., 1984; Juang and Pappa, 1985). Finally, the Kalman filter for the open-loop system can be estimated from the realized state-space model and the open-loop Kalman filter Markov parameters through a least-square approach.

With this closed-loop identification, an iterative LQG controller design can be performed. Since the Kalman filter used in this LQG controller is obtained directly from the closed-loop identification, it automatically takes into account the effect of the controller on the noise statistics. The $L Q R$ tends to reject the process noise and the Kalman filter tends to filter out the measurement noise. Therefore, the closed-loop identification can improve the LQG design and an updated LQG controller can enhance the closed-loop identification in the next cycle. After a certain number of iterations, the LQG controller will converge.

A similar approach is presented by Liu and Skelton (1990). As compared to that approach, this paper has the following contributions. First, the proposed method is developed under the stochastic framework rather than a deterministic one. Second, the Kalman filter gain is also identified so that it can be used for state estimation directly. Third, random excitation rather than pulse response is used for the closed-loop identification. Finally, since the Kalman filter gain is identified, LQR state feedback is used rather than output feedback. Numerical and experimental examples are provided to illustrate and validate this controller design.

\section{Closed-Loop State-Space and ARX Models Rela- tionship}

Since the relation between the input/output data and the model parameters of a state-space model is nonlinear, parameter estimation of a state-space model from input/output data is a nonlinear programming problem. Nonlinear programming is difficult to solve in general and involves complex iterative numerical methods. The convergence and uniqueness of the solution are also not guaranteed. Unlike a state-space model, the ARX model has a linear relationship between its model parameters and input/output data. Therefore, linear programming can be used for identifying the ARX model. After obtaining the ARX model, a state-space model can be developed based on the relation between these two models. In this section, the relation between a closed-loop state-space model and an ARX model is derived by using $z$-transforms.

A finite-dimensional, linear, discrete-time, time-invariant system can be modeled as:

$$
\begin{gathered}
x_{k+1}=A x_{k}+B u_{k}+w_{k} \\
y_{k}=C x_{k}+v_{k}
\end{gathered}
$$

where $x \in R^{n \times 1}, u \in R^{s \times 1}, y \in R^{m \times 1}$ are state, input, and output vectors, respectively; $w_{k}$ is the process noise, $v_{k}$ the measurement noise; $[A, B, C]$ are the state-space parameters. Sequences $w_{k}$ and $v_{k}$ are assumed gaussian, white, zero-mean, and stationary with covariance matrices $W$ and $V$, respectively. One can derive a steady-state filter innovation model (Haykin, 1991):

$$
\begin{gathered}
\hat{x}_{k+1}=A \hat{x}_{k}+B u_{k}+A K \epsilon_{k} \\
y_{k}=C \hat{x}_{k}+\epsilon_{k} .
\end{gathered}
$$


where $\hat{x}_{k}$ is the a priori estimated state, $K$ is the steady-state Kalman filter gain, and $\epsilon_{k}$ is the residual after filtering. The existence of $K$ is guaranteed if the system is detectable and ( $A$, $W^{1 / 2}$ ) is stabilizable (Goodwin and Sin, 1984). The advantage of using the filter innovation model in the closed-loop identification is that one can directly identify the Kalman filter gain without estimating the covariance matrices of both process and measurement noise which usually are difficult to be obtained from test data (Chen and Huang, 1994).

Similarly, any kind of dynamic output feedback controller can be modeled as:

$$
\begin{gathered}
p_{k+1}=A_{d} p_{k}+B_{d} y_{k} . \\
u_{k}=C_{d} p_{k}+D_{d} y_{k}+r_{k},
\end{gathered}
$$

where $A_{d}, B_{d}, C_{d}$, and $D_{d}$ are the system matrices of the dynamic output feedback controller, $p_{k}$ the controller state and $r_{k}$ is the open-loop input to the closed-loop system. Combining (3) to (6), the augmented closed-loop system dynamics becomes

$$
\begin{gathered}
\eta_{k+1}=A_{c} \eta_{k}+B_{c} r_{k}+A_{c} K_{c} \epsilon_{k} \\
y_{k}=C_{c} \eta_{k}+\epsilon_{k},
\end{gathered}
$$

where

$$
\begin{aligned}
& A_{c}=\left[\begin{array}{cc}
A+B D_{d} C & B C_{d} \\
B_{d} C & A_{d}
\end{array}\right], \quad B_{c}=\left[\begin{array}{l}
B \\
0
\end{array}\right], \\
& A_{c} K_{c}=\left[\begin{array}{c}
A K+B D_{d} \\
B_{d}
\end{array}\right], \quad C_{c}=\left[\begin{array}{ll}
C & 0
\end{array}\right],
\end{aligned}
$$

and $\eta_{k}=\left[\begin{array}{l}k_{k}^{k} \\ p_{k}\end{array}\right]$. It is noted that $K_{c}$ can be considered as the Kalman filter gain for the closed-loop system and the existence of the steady-state $K_{c}$ is guaranteed when the closed-loop system matrix $A_{c}$ is nonsingular. Substituting (8) into (7) yields

$$
\eta_{k+1}=\bar{A} \eta_{k}+B_{c} r_{k}+A_{c} K_{c} y_{k},
$$

where $\bar{A}=A_{c}-A_{c} K_{c} C_{c}$ and is guaranteed to be asymptotically stable because the steady-state Kalman filter gain $K_{c}$ exists. The $z$-transform of $(10)$ and $(8)$ yields

$$
\begin{gathered}
\eta(z)=(z-\bar{A})^{-1}\left(A_{c} K_{c} y(z)+B_{c} r(z)\right) \\
y(z)=C_{c} \eta(z)+\epsilon(z) .
\end{gathered}
$$

Substituting (11) into (12), one has

$$
y(z)=C_{c}(z-\bar{A})^{-1}\left(A_{c} K_{c} y(z)+B_{c} r(z)\right)+\epsilon(z) .
$$

The inverse $z$-transform of (13) with $(z-\bar{A})^{-1}=\sum_{i=1}^{\infty} \bar{A}^{i-1} z^{-i}$ yields

$$
y_{k}=\sum_{i=1}^{\infty} C_{\mathrm{c}} \bar{A}^{i-1} A_{c} K_{c} y_{k-i}+\sum_{i=1}^{\infty} C_{c} \bar{A}^{i-1} B_{c} r_{k-i}+\epsilon_{k} .
$$

Since $\bar{A}$ is asymptotically stable, $\bar{A}^{i} \approx 0$ if $i>q$ for a sufficient large number $q$. Thus (14) becomes

$$
y_{k} \approx \sum_{i=1}^{q} a_{i} y_{k-i}+\sum_{i=1}^{q} b_{i} r_{k-i}+\epsilon_{k}
$$

where

$$
a_{i}=C_{c} \bar{A}^{i-1} A_{c} K_{c}, b_{i}=C_{c} \bar{A}^{i-1} B_{c} .
$$

The model described by (15) is the ARX model which directly represents the relationship between the input and output of the closed-loop system. The coefficient matrices $a_{i}$ and $b_{i}$ can be estimated through least-square methods from random excitation input $r_{k}$ and the corresponding output $y_{k}$. From (15) by neglecting $\epsilon_{k}$, the least-square problem becomes $\xi^{T}=\left[a_{1}\right.$ $\left.\begin{array}{llll}b_{1} & \cdots & a_{q} & b_{q}\end{array}\right] \Phi^{T}$ or $\xi=\Phi \theta$, where

$$
\Phi=\left[\begin{array}{ccccccc}
y_{q}^{T} & r_{q}^{T} & y_{q-1}^{T} & r_{q-1}^{T} & \cdots & y_{1}^{T} & r_{1}^{T} \\
y_{q+1}^{T} & r_{q+1}^{T} & y_{q}^{T} & r_{q}^{T} & \cdots & y_{2}^{T} & r_{2}^{T} \\
\vdots & \vdots & \vdots & \vdots & \ddots & \vdots & \vdots \\
y_{l-1}^{T} & r_{l-1}^{T} & y_{l-2}^{T} & r_{l-2}^{T} & \cdots & y_{l-q}^{T} & r_{l-q}^{T}
\end{array}\right],
$$

$\xi=\left[\begin{array}{llll}y_{q+1} & y_{q+2} & \cdots & y_{l}\end{array}\right]^{T}, \theta=\left[\begin{array}{llllll}a_{1} & b_{1} & a_{2} & b_{2} & \cdots & a_{q}\end{array}\right.$ $\left.b_{q}\right]^{T}$, and $l$ is the number of data points. The integer $l$ has to be large enough so that the $\Phi$ matrix has more rows than columns. The batch least-square solution is

$$
\theta=\left(\Phi^{T} \Phi\right)^{-1} \Phi^{T} \xi
$$

Therefore, solving for an ARX model simply involves solving a linear programming problem involving an over determined set of equations.

\section{Markov Parameters and State-Space Realization}

In the previous section, an ARX model, which represents a closed-loop system, is identified from the input/output data through the least-square method. With the known controller dynamics, the estimated ARX model can be transformed to an open-loop state-space model by the following steps. First, the closed-loop system and Kalman filter Markov parameters are calculated from the estimated coefficient matrices of the ARX model. Second, the open-loop system and Kalman filter Markov parameters are derived from the closed-loop system Markov parameters, the closed-loop Kalman filter Markov parameters, and the known controller Markov parameters. Third, the openloop state-space model is realized by using singular-value decomposition for a Hankel matrix formed by the open-loop system Markov parameters. Finally, an open-loop Kalman filter gain is calculated from the realized state-space model and the open-loop Kalman filter Markov parameters through leastsquares.

The $z$-transform of the open-loop state-space model ( 3 ) yields

$$
\hat{x}(z)=(z-A)^{-1}(B u(z)+A K \epsilon(z)) .
$$

Substituting (18) to the $z$-transform of the output Eq. (4), one has

$$
\begin{aligned}
y(z) & =C(z-A)^{-1}(B u(z)+A K \epsilon(z))+\epsilon(z) \\
& =\sum_{k=1}^{\infty} Y(k) z^{-k} u(z)+\sum_{k=0}^{\infty} N(k) z^{-k} \epsilon(z),
\end{aligned}
$$

where $Y(k)=C A^{k-1} B$ are the open-loop system Markov parameters; $N(k)=C A^{k-1} A K$, for $k=1, \ldots, \infty$, open-loop Kalman filter Markov parameters, and $N(0)=I$ which is an identity matrix. Similariy, for the dynamic output feedback controller (5) and (6) and the closed-loop state-space model (7) and $(8)$, one can derive

$$
\begin{gathered}
u(z)=\sum_{k=0}^{\infty} Y_{d}(k) z^{-k} y(z)+r(z) \\
y(z)=\sum_{k=1}^{\infty} Y_{c}(k) z^{-k} r(z)+\sum_{k=0}^{\infty} N_{c}(k) z^{-k} \epsilon(z),
\end{gathered}
$$

where $Y_{d}(0)=D_{d}$, and $Y_{d}(k)=C_{d} A_{d}^{k-1} B_{d}$, for $k=1, \ldots, \infty$, are the controller Markov parameters; $Y_{c}(k)=C_{c} A_{c}^{k-1} B_{c}$ the closed-loop system Markov parameters; and $N_{c}(0)=I, N_{c}(k)$ $=C_{c} A_{c}^{k-1} A_{c} K_{c}$, for $k=1, \ldots, \infty$, the closed-loop Kalman filter Markov parameters.

Closed-Loop System and Kalman Filter Markov Parameters. The $z$-transform of the ARX model (15) yields

$$
\left(I-\sum_{i=1}^{q} a_{i} z^{-i}\right) y(z)=\sum_{i=1}^{q} b_{i} z^{-i} r(z)+\epsilon(z) .
$$


Applying long division to (22), one has

$$
\begin{aligned}
y(z)=\left(b_{1} z^{-1}\right. & +\left(b_{2}+a_{1} b_{1}\right) z^{-2}+\left(b_{3}+a_{1}\left(b_{2}+a_{1} b_{1}\right)\right. \\
\left.+a_{2} b_{1}\right) z^{-3} & +\ldots) r(z)+\left(1+a_{1} z^{-1}+\left(a_{1} a_{1}+a_{2}\right) z^{-2}\right. \\
& \left.+\left(a_{1}\left(a_{1} a_{1}+a_{2}\right)+a_{2} a_{1}+a_{3}\right) z^{-3}+\ldots\right) \epsilon(z) .
\end{aligned}
$$

After comparing with (21), the closed-loop system and Kalman filter Markov parameters can be recursively calculated from the estimated coefficient matrices of the ARX model,

$$
\begin{gathered}
Y_{c}(k)=b_{k}+\sum_{i=1}^{k} a_{i} Y_{c}(k-i) \\
N_{c}(k)=\sum_{i=1}^{k} a_{i} N_{c}(k-i) .
\end{gathered}
$$

It is noted that $Y_{c}(0)=0, N_{c}(0)=I$, and $a_{i}=b_{i}=0$, when $i>q$. One may obtain (23) and (24) from (16) and the definition of the Markov parameters (Phan et al., 1991; Juang et al., 1993). However, the derivation is much more complex.

Open-Loop System and Kalman Filter Markov Parameters. Next, the open-loop system and Kalman filter Markov parameters can be derived from the closed-loop system Markov parameters, the closed-loop Kalman filter Markov parameters, and the known controller Markov parameters. Substituting (20) into (19) yields

$$
\begin{aligned}
& y(z)=\left(\sum_{k=1}^{\infty} Y(k) z^{-k}\right)\left(\sum_{k=0}^{\infty} Y_{d}(k) z^{-k} y(z)\right) \\
& +\sum_{k=1}^{\infty} Y(k) z^{-k} r(z)+\sum_{k=0}^{\infty} N(k) z^{-k} \epsilon(z)=\sum_{k=1}^{\infty} \alpha_{k} z^{-k} y(z) \\
& +\sum_{k=1}^{\infty} Y(k) z^{-k} r(z)+\sum_{k=0}^{\infty} N(k) z^{-k} \epsilon(z),
\end{aligned}
$$

where $\alpha_{k}=\sum_{i=1}^{\infty} Y(i) Y_{d}(k-i)$. Rearranging (25), one has

$$
\begin{aligned}
\left(I-\sum_{k=1}^{\infty} \alpha_{k} z^{-k}\right) y(z) & \\
& =\sum_{k=1}^{\infty} Y(k) z^{-k} r(z)+\sum_{k=0}^{\infty} N(k) z^{-k} \epsilon(z) .
\end{aligned}
$$

Similarly, one can apply long division to (26), and then compare it with (21), to describe the closed-loop system Markov parameters recursively in terms of the open-loop system and the controller Markov parameters.

$$
\begin{aligned}
Y_{c}(j) & =Y(j)+\sum_{k=1}^{j} \alpha_{k} Y_{c}(j-k) \\
& =Y(j)+\sum_{k=1}^{j} \sum_{i=1}^{k} Y(i) Y_{d}(k-i) Y_{c}(j-k)
\end{aligned}
$$

And the closed-loop Kalman filter Markov parameters can be recursively expressed in terms of the open-loop system Markov parameters, the open-loop Kalman filter Markov parameters, and the controller Markov parameters as follows:

$$
\begin{aligned}
N_{c}(j) & =N(j)+\sum_{k=1}^{j} \alpha_{k} N_{c}(j-k) \\
& =N(j)+\sum_{k=1}^{j} \sum_{i=1}^{k} Y(i) Y_{d}(k-i) N_{c}(j-k) .
\end{aligned}
$$

Rearranging (27) and (28), one has

$$
\begin{gathered}
Y(j)=Y_{c}(j)-\sum_{k=1}^{j} \sum_{i=1}^{k} Y(i) Y_{d}(k-i) Y_{c}(j-k) \\
N(j)=N_{c}(j)-\sum_{k=1}^{j} \sum_{i=1}^{k} Y(i) Y_{d}(k-i) N_{c}(j-k) .
\end{gathered}
$$

Equations (29) and (30) show that one can recursively calculate the open-loop system and Kalman filter Markov parameters from the closed-loop system Markov parameters in (23), the closed-loop Kalman filter Markov parameters in (24), and the known controller Markov parameters $Y_{d}(k)=C_{d} A_{d}^{k-1} B_{d}$. It is noted that $Y_{c}(0)=0$ and $N_{c}(0)=I$. One can easily verify (29) and (30) from (9), and also from the definition of the Markov parameters.

State-Space Realization. The open-loop state-space model can be realized from the open-loop system Markov parameters through the Singular Value Decomposition (SVD) method (Chen, 1984; Juang and Pappa, 1985). The first step is to form a Hankel matrix from the open-loop system Markov parameters,

$$
H(j)=\left[\begin{array}{cccc}
Y(j) & Y(j+1) & \cdots & Y(j+\beta) \\
Y(j+1) & Y(j+2) & \cdots & Y(j+\beta+1) \\
\vdots & \vdots & \ddots & \vdots \\
Y(j+\gamma) & Y(j+\gamma+1) & \cdots & Y(j+\gamma+\beta)
\end{array}\right]
$$

where $Y(j)$ is the $j$-th Markov parameter. For a noise free system, if the arbitrary integers $\beta \geq n$, and $\gamma \geq n$ (the order of the system), the Hankel matrix $H(j)$ is of rank $n$. From the measurement Hankel matrix, the realization uses the SVD of $H(1), H(1)=U \Sigma V^{T}$, to identify a $n$-th order discrete statespace model as

$$
\begin{gathered}
A=\Sigma_{n}^{-1 / 2} U_{n}^{T} H(2) V_{n} \Sigma_{n}^{-1 / 2}, \\
B=\Sigma_{n}^{1 / 2} V_{n}^{T} E_{s}, C=E_{m}^{T} U_{n} \Sigma_{n}^{1 / 2}
\end{gathered}
$$

where matrix $\Sigma_{n}$ is the upper left hand $n \times n$ partition of $\Sigma$ containing the $n$ largest singular values along the diagonal. Matrices $U_{n}$ and $V_{n}$ are obtained from $U$ and $V$ by retaining only the $n$ columns of singular vectors associated with the $n$ singular values. Matrix $E_{m}$ is a matrix of appropriate dimension having $m$ columns, all zero except that the top $m \times m$ partition is an identity matrix. $E_{s}$ is defined similarly.

Open-Loop Kaiman Filter Gain. Once the open-loop $A$ and $C$ are obtained, one can easily calculate the open-loop Kalman filter gain from the open-loop Kalman filter Markov parameters $N(k)=C A^{k} K$ in a least-square sense as follows

$$
K=\left(O^{T} O\right)^{-1} O^{T}\left[\begin{array}{c}
N(1) \\
\vdots \\
N(k)
\end{array}\right] \text {, where } O=\left[\begin{array}{c}
C A \\
\vdots \\
C A^{k}
\end{array}\right]
$$

The integer $k$ has to be large enough so that the matrix $O$ has more rows than columns. The identified Kalman filter gain can be used directly for state estimation.

\section{Iterative LQG Controller Design}

Classical LQG controllers are designed by solving two separate, but dual problems: the $L Q R$ design and Kalman filter design. Here, the Kalman filter gain can be simultaneously obtained with the open-loop state-space model through the closedloop identification. Only the LQR design based on the identified open-loop model needs to be solved. The performance index for the LQR problem is defined as

$$
\text { P. I. }=\sum_{k=1}^{\infty} y_{k}^{T} Q y_{k}+u_{k}^{T} R u_{k}=\sum_{k=1}^{\infty} x_{k}^{T} C^{T} Q C x_{k}+u_{k}^{T} R u_{k}
$$

where weighting matrices $Q$ and $R$ are design parameters. We can summarize the iterative LQG controller design as follows:

1. Use the a priori open-loop model and arbitrary covariance matrices of the measurement and process noise to design the LQR and Kalman filter. Then, calculate the controller Mar- 


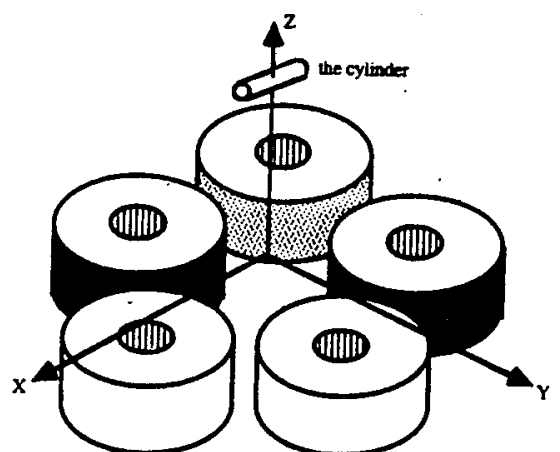

Fig. 1 Large-angle magnetic suspension test facility (LAMSTF) configuration

kov parameters. The weighting matrices $Q$ and $R$ for the $\mathrm{LQR}$ chosen here will remain the same in the following iterations.

2. Apply random excitation input to the closed-loop system and record the closed-loop input/output data.

3. Estimate the coefficient matrices of the closed-loop ARX model by using (17).

4. Calculate the closed-loop system and Kalman filter Markov parameters by using (23) and (24).

5. Calculate the open-loop system and Kalman filter Markov parameters by using (29) and (30).

6. Realize the open-loop state-space system matrices $[\hat{A}$, $\hat{B}, \hat{C}]$ by using (31) and (32).

7. Estimate the open-loop Kalman filter gain $\hat{K}$ by using (33).

8. Obtain the LQR feedback gain $F$ by solving the corresponding Riccati equation based on the identified open-loop model.

9. Form the updated LQG controller in (5) and (6) by using $A_{d}=\hat{A}-\hat{B} F-\hat{A} \hat{K} \hat{C}, B_{d}=\hat{A} \hat{K}, C_{d}=-F$, and $D_{d}=0$.

10. Calculate the updated controller Markov parameters and check the convergence of the controller by

$$
\delta=\sum_{k=0}^{n} \| Y_{d}(k)_{\text {updered }}-Y_{d}(k)_{\text {previous } \|_{2}} \text {. }
$$

If $\delta$ is greater than a desired value, go back to step 2, otherwise stop.

\section{Numerical and Experimental Example}

The proposed iterative LQG controller design has been applied to control design of the Large-Angle Magnetic Suspension Test Facility (LAMSTF) (Groom and Britcher, 1992; Groom and Schaffner, 1990) developed in NASA Langley Research Center (see Fig. 1). The LAMSTF is a laboratory-scale research project to demonstrate the magnetic suspension of objects over wide ranges of attitudes. This system represents a scaled model of a planned Large-Gap Magnetic Suspension System. The LAMSTF system consists of a planar array of five copper electromagnets which actively suspend a small cylinder with a permanent magnet core. The cylinder is a rigid body and has six independent degrees of freedom, namely, three displacements $(x, y$, and $z$ ) and rotations (pitch, yaw, and roll). Currents in the electromagnets generate a magnetic field which produces a net force and torque on the suspended cylinder. The roll of the cylinder is uncontrollable, and is assumed to be motionless. Five pairs of the LEDs and light receivers are used to indirectly sense the pitch and yaw angles, and three displacements of the cylinder's centroid. Therefore, the control inputs to the system consist of five currents sent into five electromagnets and the system outputs are five voltage signais measured from five photo detectors. The forces on the cylinder are, in general, nonlinear functions of space and current. Therefore, only the linear time-invariant perturbed motion about an equilibrium state is
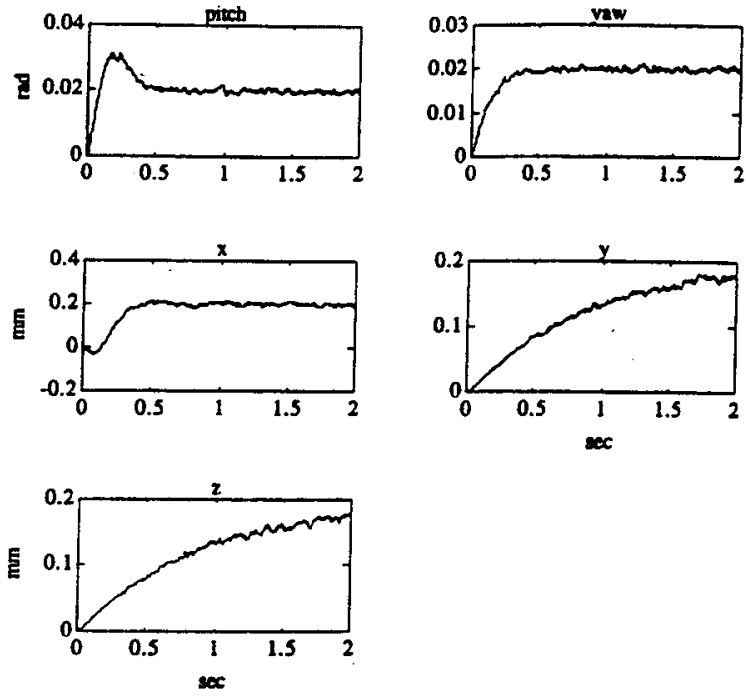

Fig. 2 Simulated step response with the initial LQG controller

considered. Because it is difficult to accurately model the magnetic field and its gradients, the analytical model contains some modeling errors. Therefore, the performance of the LQG controller based on the analytical model alone is unsatisfactory.

The system matrices of the analytical model are shown in the appendix. The eigenvalues of the system matrix indicate that the LAMSTF system includes highly unstable real poles (about $10 \mathrm{~Hz}$ ) and low-frequency oscillatory modes (about 0.16 $\mathrm{Hz}$ ). For both numerical simulation and experiment, the sampling rate is $250 \mathrm{~Hz}$. The performance index used for the LQR design is also shown in the Appendix. The step command for all simulations and experiments is 0.02 radian for pitch and yaw, and $0.2 \mathrm{~mm}$ for $x, y$, and $z$.

In the numerical simulation, the analytical model is used as the true model. In each iteration, the ratios of the process and measurement noise to the corresponding signal are 2 and 1 percent, respectively. To simulate modeling error and unknown noise statistics, the initial LQG controller is designed by using an altered model of which each parameter is 5 percent greater than the corresponding parameter of the analytical model and guessed covariance matrices of noise $W=10 I_{10 \times 10}$ and $V=$ $I_{5 \times 5}$. The simulated step response with this initial controller for the pitch, yaw, $x, y$, and $z$ is shown in Fig. 2. It is clear that
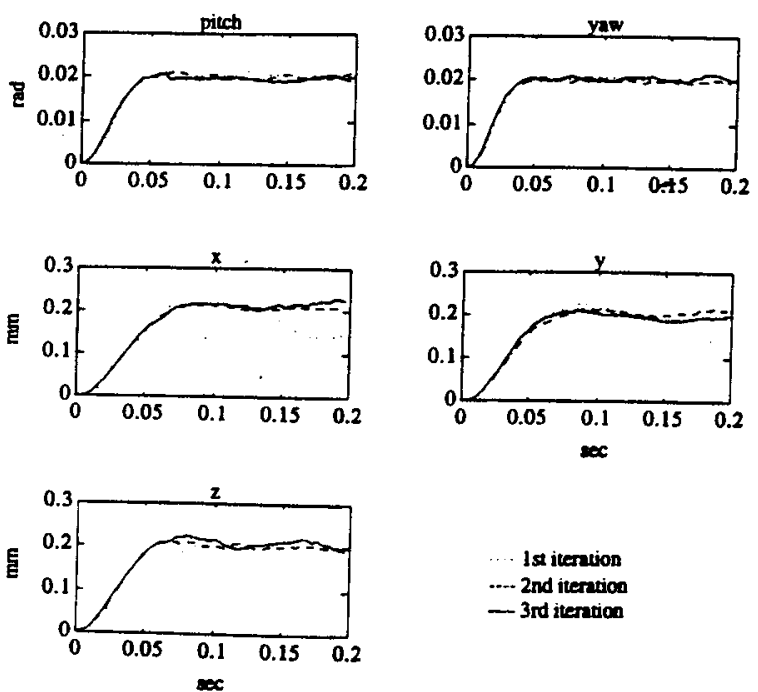

Fig. 3 Comparison of the simulated step response with the iterative LQG controller 
the result is very poor. After performing the first iteration of the proposed iterative LQG controller design, the step response shown in Fig. 3 is greatly improved. The performance is further improved slightly in the following iterations. Figure 4 shows how the controller converges by comparing the $(1,1)$ element of the controller Markov parameters.

For a noise free system, the exact open-loop model can be obtained after the first closed-loop identification and no further iteration is required. In this case, the identified Kalman filter gain becomes the dead-beat observer gain (Phan et al., 1991; Juang et al., 1993). For a noise corrupted system, iterations are required to update the open-loop model and the Kalman filter gain until the iterative LQG controller converges. Although the numerical simulations show that the iterative controller can converge quickly, the required conditions to guarantee the convergence need further study.

In the experiments, the analytical model and guessed covariance matrices of noise $W=10 I_{10 \times 10}$ and $V=I_{5 \times 5}$ are used to design the initial LQG controller. The experimental step response with this initial controller is also very poor. The experimental step responses for the first three iterations are compared in Fig. 5 to demonstrate how the step response is improved with iteration. In each iteration, the open-loop system model and the Kalman filter gain are updated through the closed-loop identification from experimental data. The experimental step response improves with each iteration, similar to the simulated cases. The experimental steady-state errors, however, do not go to zero in each case. This is due to drift in the sensor zero between experiments. The system's dynamics have been found to be insensitive to these small changes in the operating point. The results show that the proposed iterative LQG controller design is very effective for controlling this highly unstable magnetic suspension system.

\section{Conclusion}

In contrast to most existing LQG controller designs of which the great majority solve two separate, but dual problems: the LQR and Kalman filter design, this paper proposes an iterative LQG controller design approach. A closed-loop identification method is developed to update the open-loop state-space model and the Kalman filter gain simultaneously from the closedloop input/output test data. The method is derived under the stochastic framework, taking into account the effects of process noise as well as measurement noise. For a noise free system, the exact open-loop model can be obtained after the first closedloop identification and the identified Kalman filter gain becomes the dead-beat observer gain. For a noise corrupted system, iterations are required to update the open-loop model and the Kalman filter gain from testing until the iterative LQG controller converges. In each iteration, since the Kalman filter gain is identified directly from test data, the LQG design is simplified to be an LQR design. A highly unstable large-angle magnetic

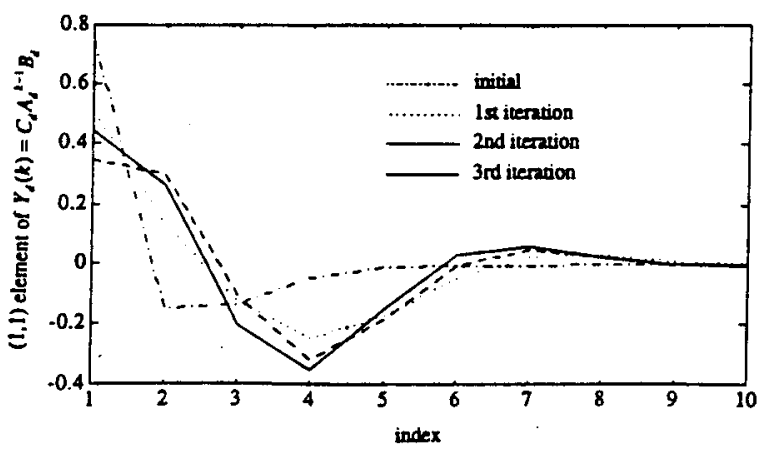

Fig. 4 Comparison of the $(1,1)$ element of the controller Markov parameters $Y_{d}(k)=C_{d} A_{d}^{k-1} B_{d}$
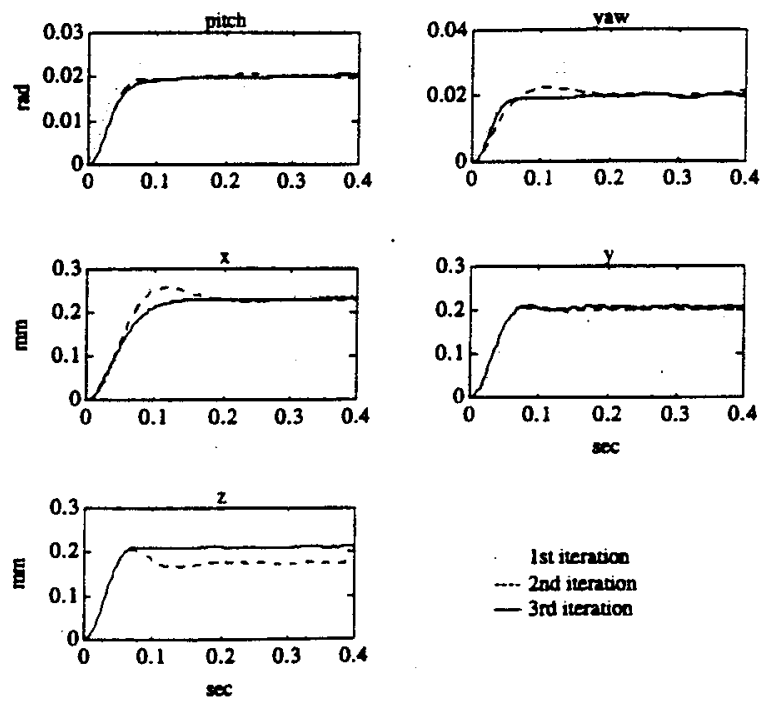

Fig. 5 Comparison of the testing step response with the iterative LQG controller

suspension system is used to validate this controller design. Both numerical simulations and test data show that the controller converges quickly and is very effective when the system is subjected to modeling error and unknown noise statistics.

\section{Acknowledgment}

This work was supported partially by NASA Langley Research Center (NAG1-1056). This support is gratefully acknowledged.

\section{References}

Chen, C. T.; 1984, Linear System Theory and Design. Second Edition. Chapter 6, CBS College Publishing. New York.

Chen. C.-W.. Huang, J.-K., Phan, M., and Juang, J.-N., 1992a, "Integrated System Identification and Modal State Estimation for Control of Flexible Space Structures," AIAA J. Guidance, Control and Dynamics. Vol. 15, No. 1, pp. 8895.

Chen, C.-W., Huang. J.-K., and Juang. J.-N.. 1992b, "Identification of Linear Stochastic Systems Through Projection Filters," Proc. AlAA Structures. Structural Dynamics and Materials Conf., pp. 2330-2340, also to appear in AIAA J. Guidance, Control and Dynamics.

Chen, C.-W.. Juang, J.-N.. and Huang. J.-K., 1993, "Adaptive Linear System Identification and State Estimation." Control and Dynamic Systems: Advances in Theory and Applications, Vol. 57. Multidisciplinary Engineering Systems: Design and Optimization Techniques and Their Application, C. T. Leondes, ed., Academic Press, pp. 331-368.

Chen, C.-W., and Huang, J.-K., 1994, "Estimation of Steady-State Optimal Filter Gain from Non-Optimal Kalman Filter Residuals," ASME JouRNAL OF DYNAMIC SYST., MEAs., AND CONTR., Vol. 116. No. 3, pp. 550-553.

Goodwin, G. C., and Sin. K. S., 1984, Adaptive Filtering. Prediction and Control. Prentice-Hall. Englewood Cliffs, NJ 07632.

Groom, N. J., and Britcher, C. P., 1992, "Open-Loop Characteristics of Magnetic Suspension Systems Using Electromagnets Mounted in a Planar Array," NASA-TP 3229.

Groom, N. J., and Schaffner. R. P., 1990, "An LQR Controller Design Approach for a Large Gap Magnetic Suspension System (LGMSS)," NASA TM101606.

Haykin, S., 1991, Adaptive Filter Theory, Second edition, Prentice-Hall, Englewood Cliffs. NJ.

Juang, J.-N., and Pappa, R. S., 1985, “An Eigensystem Realization Algorithm for Modal Parameter Identification and Model Reduction," AIAA J. Guidance, Control, and Dynamics, Vol. 8, pp. 620-627.

Juang, J.-N.. Phan. M., Horta, L. G.. and Longman, R. W., 1993, "Identification of Observer/Kalman Filter Markov Parameters: Theory and Experiments," AIAA J. Guidance, Control and Dynamics, Vol. 16, No. 2, pp. 320-329.

Liu, K., and Skeiton. R. E., 1990, "Closed-Loop Identification and Iterative Controller Design," in Proc. IEEE Int. Conf. Decision and Control, pp. 482487.

Phan, M., Horta, L. G., Juang, J.-N., and Longman, R. W., 1993. "Linear System Identification via an Asymptotically Stable Observer," J. Optimization Theory and Applicarion, Vol. 79, No. 1, 1993, pp. 59-86.

Phan, M., Juang, J.-N., Horta, L. G., and Longman. R. W., 1992. “'System Identification from Closed-Loop Data with Known Output Feedback Dynamics," J. Guidance, Control and Dynamics, Vol. 17, No. 4, 1994, pp. 661-669. 


\section{A P P E N D I X}

The analytical model of the large-angle magnetic suspension test facility is

$$
\begin{gathered}
\dot{x}=A_{m} x+B_{m} u \\
y=C_{m} x
\end{gathered}
$$

where $x=\left[\begin{array}{l}x_{p} \\ x_{p}\end{array}\right], A_{m}=\left[\begin{array}{ll}0_{s \times s} & l_{s \times 5} \\ A_{21} & A_{22}\end{array}\right], B_{m}=\left[\begin{array}{l}0_{3 \times s} \\ B_{2}\end{array}\right]$ and $C_{m}=\left[C_{1}\right.$ $\left.0_{5 \times s}\right]$. The state variable $x_{p}$ includes pitch and yaw angles and three linear displacements of the cylinder's centroid. The matrices $A_{21}, A_{22}, B_{2}$, and $C_{1}$ are
The eigenvalues of the system matrix $A_{m}$ are $\pm 58.78, \pm 57.81$, $\pm 9.78, \pm j 7.97$, and $\pm j 0.96$. The matrix $C_{1}$ which relates the sensor output voltage to the displacement can be obtained from calibration and is assumed known. To recover the displacement from the sensor output voltage, one can use $x_{p}=C_{1}^{-1} y$.

The performance index for the state feedback design is chosen as

$$
P . I .=\sum_{k=1}^{\infty} y_{k}^{T} Q y_{k}+u_{k}^{T} R u_{k}
$$

where $Q=\left(C_{1}^{-1}\right)^{T} \operatorname{diag}\left[\begin{array}{lllll}1 . e 3 & 1 . e 3 & 2 . e 8 & 2 . e 8 & 2 . e 8\end{array}\right] C_{1}^{-1}$ and $R$ $=I_{5 \times 5}$.

$$
A_{21}=\left[\begin{array}{ccccc}
3.3415 e+03 & 0 & -3.9392 e+04 & 4.9534 e-12 & 2.0811 e-12 \\
0 & 3.3415 e+03 & -4.9534 e-12 & 4.8609 e-12 & -1.4472 e-11 \\
-9.8070 e+00 & -2.4664 e-15 & 4.9937 e+01 & 4.3604 e-15 & -2.5089 e-02 \\
-3.6031 e-15 & 1.9618 e-15 & 4.3604 e-15 & 9.5577 e+01 & -9.0007 e-15 \\
-2.3357 e-16 & -3.6031 e-15 & -2.5089 e-02 & -9.0007 e-15 & -9.1324 e-01
\end{array}\right],
$$

$A_{22}=0_{5 \times 5}$,

$$
B_{2}=\left[\begin{array}{ccccc}
3.8370 e+01 & 3.8370 e+01 & 3.8370 e+01 & 3.8370 e+01 & 3.8370 e+01 \\
0 & 8.9802 e+01 & 5.5514 e+01 & -5.5514 e+01 & -8.9802 e+01 \\
2.2144 e-01 & -1.5274 e-01 & 7.8453 e-02 & 7.8453 e-02 & -1.5274 e-01 \\
0 & 1.2154 e-01 & -1.9674 e-01 & 1.9674 e-01 & -1.2154 e-01 \\
-2.7672 e-01 & -8.5465 e-02 & 2.2388 e-01 & 2.2388 e-01 & -8.5465 e-02
\end{array}\right] \text {, }
$$

$C_{1}=\left[\begin{array}{ccccc}8.9024 e+01 & 0 & 0 & 0 & 6.0976 e+03 \\ 0 & 0 & 7.8740 e+03 & 0 & 0 \\ -1.1625 e+02 & 0 & 0 & 0 & 6.2500 e+03 \\ 0 & 9.5425 e+01 & 0 & -6.5359 e+03 & 0 \\ 0 & -1.0725 e+02 & 0 & -5.1813 e+03 & 0\end{array}\right]$. 


\section{Engineering Notes}

ENGINEERING NOTES are short manuscripts describing new developments or important results of a preliminary nature. These Notes cannot exceed 6 manuscript pages and 3 figures; a page of text may be substituted for a figure and vice versa. After informal review by the editors, they may be published within a few months of the date of receipt. Style requirements are the same as for regular contributions (see inside back cover).

\section{State-Space System Identification from Closed-Loop Frequency Response Data}

\author{
Jen-Kuang Huang, ${ }^{*}$ Hyun Chang Lee, ${ }^{\dagger}$ \\ and Marco P. Schoen ${ }^{\dagger}$ \\ Old Dominion University, \\ Norfolk, Virginia 23529-0247 \\ and \\ Min-Hung Hsiao \\ Taipei Institute of Technology, Taipei 10643, Taiwan
}

\section{Introduction}

$\mathbf{E}$ XTENSIVE research has been conducted in active control of dynamic systems. Achieving high control performance on these systems usually requires an accurate model. Such a model can be derived from system identification techniques using experimental data. Recently, a method was developed to identify a state-space model from frequency response data for open-loop systems by using the state-space frequency domain identification algorithm.' This method uses a matrix-fraction for the curve fitting, and the curve fitting is reformulated as a linear problem that can be solved by the ordinary least-squares method in one step.

A different method has been proposed to identify a state-space plant from closed-loop I/O time-domain data with known feedback dynamics. ${ }^{2}$ This Note is an extension of this time-domain closedloop identification method to frequency domain. The method can identify a linear open-loop stochastic system from closed-loop frequency response test data with known feedback dynamics. The relationship between the frequency response function (FRF) and the closed-loop system and Kalman-filter Markov parameters is derived for linear stochastic systems. Once the closed-loop system and Kalman-filter Markov parameters are obtained from FRF, a recursive formula for computing the open-loop system and the Kalmanfilter Markov parameters from the closed-loop system, Kalman filter and controller Markov parameters can be used. Finally, the openloop system can be realized from the calculated open-loop system Markov parameters.

\section{Linear State-Space and FRF Relationship}

A finite-dimensional, linear, discrete-time, time-invariant system can be modeled as

$$
\begin{gathered}
x_{k+1}=A x_{k}+B u_{k}+w_{k} \\
y_{k}=C x_{k}+v_{k}
\end{gathered}
$$

where $x \in R^{n \times 1}, u \in R^{s \times 1}, y \in R^{m \times 1}$ are state, input, and output vectors, respectively; $w_{k}$ is the process noise; $v_{k}$ is the measurement

Received Feb. 27, 1995; revision received April 30, 1996; accepted for publication June 28, 1996. Copyright (C) 1996 by the American Institute of Aeronautics and Astronautics. Inc. All rights reserved.

- Professor, Department of Mechanical Engineering. Member AIAA.

${ }^{\dagger}$ Ph.D. Student, Department of Mechanical Engineering.

${ }^{\ddagger}$ Associate Professor. Department of Mechanical Engineering. noise and $[A, B, C]$ are the state-space parameters. Sequences $w_{k}$ and $v_{k}$ are assumed Gaussian, white, and stationary with zero mean and covariance matrices $Q$ and $R$, respectively. One can derive 3 steady-state filter innovation model ${ }^{\text {l: }}$ :

$$
\begin{gathered}
\hat{x}_{k+1}=A \hat{x}_{k}+B u_{k}+A K \varepsilon_{k} \\
y_{k}=C \hat{x}_{k}+\varepsilon_{k}
\end{gathered}
$$

where $\hat{x}_{k}$ is the a priori estimated state, $K$ is the steady-state Kalmanfilter gain, and $\varepsilon_{k}$ is the residual after filtering: $\varepsilon_{k}=y_{k}-C \dot{x}_{k}$. On the other hand, a dynamic output feedback controller can be modeled as

$$
\begin{gathered}
p_{k+1}=A_{d} p_{k}+B_{d} y_{k} \\
u_{k}=C_{d} p_{k}+D_{d} y_{k}+r_{k}
\end{gathered}
$$

where $A_{d}, B_{d}, C_{d}$, and $D_{d}$ are the system matrices of the controller, $p \in R^{l \times 1}$ is the controller state vector; and $r \in R^{s \times 1}$ is the reference input to the closed-loop system. Combining Eqs. (3) and (6), the augmented closed-loop system dynamics become

$$
\begin{gathered}
\eta_{k+1}=A_{c} \eta_{k}+B_{c} r_{k}+A_{c} K_{c} \varepsilon_{k} \\
y_{k}=C_{c} \eta_{k}+\varepsilon_{k}
\end{gathered}
$$

or

$$
\eta_{k+1}=\bar{A} \eta_{k}+B_{c} r_{k}+A_{c} K_{c} y_{k}
$$

where

$$
\begin{array}{cc}
\eta_{k}=\left[\begin{array}{l}
\hat{x}_{k} \\
p_{k}
\end{array}\right], \quad A_{c}=\left[\begin{array}{cc}
A+B D_{d} C & B C_{d} \\
B_{d} C & A_{d}
\end{array}\right] \\
B_{c}=\left[\begin{array}{l}
B \\
0
\end{array}\right], & A_{c} K_{c}=\left[\begin{array}{c}
A K+B D_{d} \\
B_{d}
\end{array}\right] \\
C_{c}=\left[\begin{array}{ll}
C & 0
\end{array}\right], \quad \text { and } \quad \bar{A}=A_{c}-A_{c} K_{c} C_{c}
\end{array}
$$

The $z$ transforms of Eqs. (8) and (9) yield

$$
\begin{gathered}
y(z)=C_{c} \eta(z)+\varepsilon(z) \\
\eta(z)=\left(z I_{t}-\bar{A}\right)^{-1}\left[A_{c} K_{c} y(z)+B_{c} r(z)\right]
\end{gathered}
$$

where $I_{t}$ is an identity matrix with dimension $t=n+l$. Substituting Eq. (11) into Eq. (10), one obtains

$$
\begin{aligned}
& y(z)=\left[I_{m}-C_{c}\left(z I_{t}-\bar{A}\right)^{-1} A_{c} K_{c}\right]^{-1} C_{c}\left(z I_{t}-\bar{A}\right)^{-1} B_{c} r(z) \\
& \quad+\left[I_{m}-C_{c}\left(z I_{t}-\bar{A}\right)^{-1} A_{c} K_{c}\right]^{-1} \varepsilon(z)
\end{aligned}
$$

The $z$ transforms of the dynamic output feedback controller (5) and (6) and the closed-loop state-space model (7) and (8) yield

$$
\begin{gathered}
u(z)=\sum_{k=0}^{\infty} Y_{d}(k) z^{-k} y(z)+r(z) \\
y(z)=\sum_{k=1}^{\infty} Y_{c}(k) z^{-k} r(z)+\sum_{k=1}^{\infty} N_{c}(k) z^{-k} \varepsilon(z)
\end{gathered}
$$


where $Y_{d}(k)=C_{d} A_{d}^{k-1} B_{d}$ are the controller Markov parameters, $Y_{c}(k)=C_{c} A_{c}^{k-1} B_{c}$ are the closed-loop-system Markov parameters, and $N_{c}(k)=C_{c} A_{c}^{k-1} A_{c} K_{c}$ are the closed-loop Kalman-filter Markov parameters. Note also that $Y_{d}(0)=D_{d}$ and $N_{c}(0)=I_{m}$.

The transfer-function matrix of the system described by Eqs. (12) and (14) is

$$
\begin{aligned}
& G\left(z^{-1}\right)\left[I_{m}-C_{c}\left(z I_{t}-\bar{A}\right)^{-1} A_{c} K_{c}\right]^{-1} C_{c}\left(z I_{t}-\bar{A}\right)^{-1} B_{c} \\
& \quad=\sum_{k=1}^{\infty} Y_{c}(k) z^{-k}
\end{aligned}
$$

The FRF is simply the transfer function matrix $G\left(z^{-1}\right)$ calculated along the unit circle in the $z$ plane. It is also chosen that the transfer function matrix can be expressed by a left-fraction description ${ }^{1}$ as

$$
G\left(z^{-1}\right)=\alpha^{-1}\left(z^{-1}\right) \beta\left(z^{-1}\right)
$$

where both $\alpha\left(z^{-1}\right)$ and $\beta\left(z^{-1}\right)$ are matrix polynomials and can be found as a solution of the least-squares method. From Eq. (7.19) in Ref. 1, one has

$$
\begin{aligned}
\left(\sum_{i=0}^{p} \alpha_{i} z^{-i}\right)\left[\sum_{i=1}^{\infty} Y_{c}(i) z^{-i}\right]= & \sum_{i=0}^{p} \beta_{i} z^{-i} \\
& \alpha_{0}=I_{m}, \quad \beta_{0}=0
\end{aligned}
$$

From this relation, the closed-loop-system Markov parameters can be recursively calculated from the estimated $\alpha$ and $\beta$ matrix polynomials by using the parameter convolution of polynomial products as follows:

$$
Y_{c}(k)=\beta_{k}-\sum_{i=1}^{k} \alpha_{i} Y_{c}(k-i)
$$

Similarly, the closed-loop Kalman-filter Markov parameters can be recursively calculated from the estimated $\alpha$ matrix polynomials as follows:

$$
N_{c}(k)=-\sum_{i=1}^{k} \alpha_{i} N_{c}(k-i)
$$

Then, from the closed-loop-system Markov parameters $Y_{c}(k)$ and the closed-loop Kalman-filter Markov parameters $N_{c}(k)$, one can recursively calculate ${ }^{2}$ the open-loop-system Markov parameters $Y(k)=C A^{k-1} B$ and the open-loop Kalman-filter Markov parameters $N(k)=C A^{k-1} A K$ with the known controller Markov parameters $Y_{d}(k)=C_{d} A_{d}^{k-1} B_{d}$

$$
\begin{gathered}
Y(j)=Y_{c}(j)-\sum_{k=1}^{j} \sum_{i=1}^{k} Y(i) Y_{d}(k-i) Y_{c}(j-k) \\
N(j)=N_{c}(j)-\sum_{k=1}^{j} \sum_{i=1}^{k} Y(i) Y_{d}(k-i) N_{c}(j-k)
\end{gathered}
$$

Note that $Y_{d}(0)=D_{d}, N_{c}(0)=I_{m}$, and $Y_{c}(0)=\beta_{0}$. The openloop state-space model can be realized from the open-loop-system Markov parameters through the singular value decomposition method.' ' Once the open-loop $A$ and $C$ are obtained, one can easily calculate the open-loop Kalman filter gain from the openloop Kalman-filter Markov parameters $N(k)$ in a least-squares sense. ${ }^{2}$

\section{Numerical and Test Example}

An example is provided that consists of numerical simulations and actual hardware tests to validate the feasibility of the proposed frequency-domain closed-loop identification method. The large-gap magnetic suspension system ${ }^{2}$ consists of a planar array of five copper electromagnets that actively suspend a small cylinder with a permanent magnet core. The cylinder is a rigid body and has six independent degrees of freedom. namely, three displacements $(x, y$,
Table 1 Comparison of eigenvalues of discrete-time analytical and identified model

\begin{tabular}{lcc}
\hline \hline & $\begin{array}{c}\text { Identified } \\
\text { from simulation } \\
\text { (1\% noise variance) }\end{array}$ & $\begin{array}{c}\text { Identified } \\
\text { from testing }\end{array}$ \\
\hline 1.1687 & 1.1686 & 1.2892 \\
1.1629 & 1.1595 & 1.2796 \\
1.0101 & 1.0019 & 1.0327 \\
0.9810 & 0.9794 & 0.9042 \\
$0.9977 \pm 0.0257 i$ & $0.9051 \pm 0.0983 i$ & $1.0094 \pm 0.0341 i$ \\
$0.9920 \pm 0.0133 i$ & $0.8546 \pm 0.1947 i$ & $0.9972 \pm 0.0221 i$ \\
$0.8633 \pm 0.0009 i$ & $0.8749,0.9323$ & $0.8451 \pm 0.2084 i$ \\
\hline
\end{tabular}
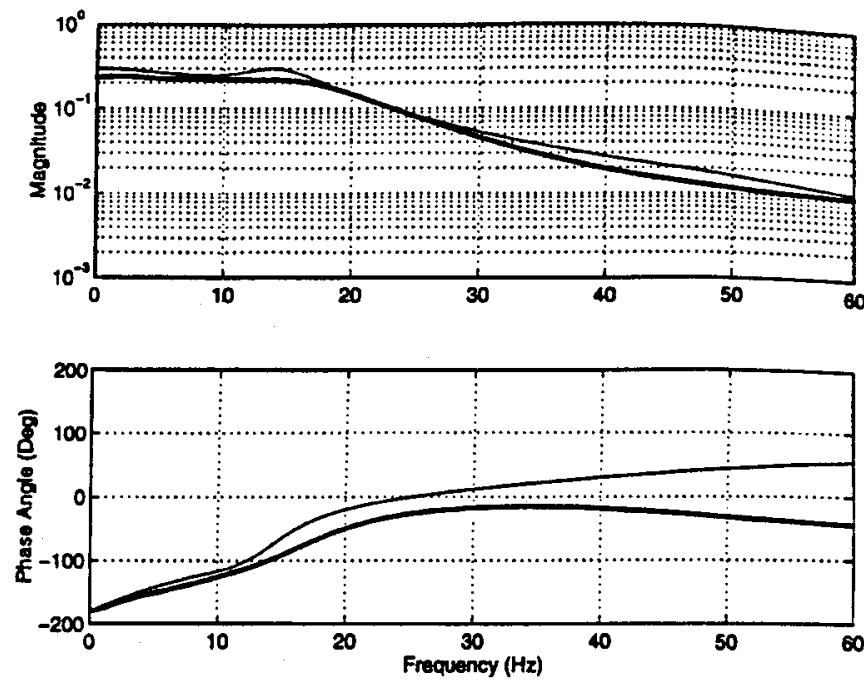

Fig. 1 Comparison of the closed-loop analytical (thin line) and reconstructed (thick line) input-1/output-1 FRFs. The reconstructed FRF is obtained using the identified system matrices.

and $z$ ) and rotations (pitch, yaw, and roll). The roll of the cylinder is uncontrollable, and is assumed to be motionless. Because it is difficult to accurately model the magnetic field and its gradients, the analytical model needs to be improved through identification from experimental data. The discrete-time state-space parameters of the system and the dynamic output feedback using a sampling rate of $250 \mathrm{~Hz}$ are shown in the Appendix.

Table 1 compares identified system eigenvalues with true ones from numerical simulations. The results show perfect match when there is no noise and quite good agreement even with $1 \%$ of processing and measurement noises ( $1 \%$ noise variance). Figure 1 also shows the comparison of the closed-loop analytical (thin line) and reconstructed (thick line) input-1/output-1 FRFs.

Five experiments also were performed. In each experiment, only one of the five actuators had a single random reference input, and all others had zero reference input. A total of 4096 data points at a sampling rate of $250 \mathrm{~Hz}$ from each sensor were recorded. Six FRFs from these single input/six output data can be derived. The experiment is repeated by sending the same single random input to a different actuator each time. Thirty FRFs were obtained. The order of the matrix polynomial was set to 13 . The identified eigenvalues from testing are shown in Table 1.

\section{Concluding Remarks}

A method of identifying a linear state-space model of a plant from closed-loop frequency response data with known feedback dynamics is developed. The main contribution is the derivation of the relationship between the open-loop-system Markov parameters and the closed-loop frequency response function for a linear stochastic system. Numerical simulations and experimental results of a highly unstable large-gap magnetic suspension system are presented. 
Appendix: Analytical System and Controller Matrices

The matrices of Eqs. (1), (2), (5), and (6) are as follows:

$$
\begin{aligned}
& A=\left[\begin{array}{rrrrrrrrrr}
1.1687 & 0.0006 & -0.0000 & 0.0000 & 0.0000 & 0.0000 & 0.0000 & -0.0000 & -0.0000 & -0.0000 \\
-0.0000 & 1.1629 & -0.0000 & -0.0000 & -0.0000 & -0.0000 & 0.0000 & -0.0000 & 0.0000 & -0.0000 \\
-0.0000 & 0.0001 & 1.0178 & -0.0017 & -0.0037 & 0.0021 & 0.0074 & -0.0127 & 0.0112 & 0.0006 \\
-0.0000 & 0.0000 & 0.0001 & 1.0051 & 0.0001 & 0.0295 & 0.0006 & 0.0015 & -0.0011 & 0.0003 \\
0.0000 & 0.0002 & -0.0004 & 0.0008 & 1.0106 & -0.0018 & 0.0223 & 0.0066 & -0.0039 & 0.0030 \\
0.0000 & -0.0000 & -0.0021 & -0.0240 & 0.0005 & 0.9908 & 0.0028 & -0.0010 & 0.0003 & -0.0011 \\
0.0000 & -0.0001 & -0.0064 & -0.0001 & -0.0213 & -0.0041 & 0.9692 & 0.0064 & 0.0004 & 0.0003 \\
-0.0000 & -0.0000 & 0.0109 & -0.0009 & -0.0045 & 0.0021 & 0.0050 & 0.9260 & -0.0549 & 0.0028 \\
0.0000 & -0.0000 & -0.0086 & 0.0009 & 0.0032 & 0.0009 & 0.0031 & -0.0589 & 0.9125 & -0.0008 \\
0.0000 & -0.0000 & 0.0004 & 0.0002 & 0.0006 & 0.0012 & 0.0545 & -0.0002 & -0.0002 & 0.8652
\end{array}\right] \\
& B=\left[\begin{array}{rrrrr}
0.0035 & 0.0706 & 0.0519 & -0.0363 & -0.0633 \\
-0.0434 & -0.0326 & -0.0340 & -0.0425 & -0.0396 \\
0.0580 & -0.0454 & 0.0983 & -0.0361 & 0.0254 \\
-0.0926 & -0.0315 & 0.0881 & 0.0865 & -0.0218 \\
0.1160 & 0.0124 & 0.0263 & 0.0982 & -0.0242 \\
-0.1015 & -0.0368 & 0.1033 & 0.0854 & -0.0154 \\
0.1373 & 0.0057 & 0.0719 & 0.0859 & -0.0066 \\
-0.0159 & -0.0637 & -0.1326 & 0.1165 & 0.0625 \\
0.0158 & -0.1531 & -0.0261 & 0.0041 & 0.1245 \\
-0.0484 & -0.0800 & -0.0513 & -0.0553 & -0.1009
\end{array}\right] \\
& C=\left[\begin{array}{rrrrrrrrrr}
-0.0313 & 0.4029 & -0.0469 & 0.2269 & -0.0381 & -0.1961 & 0.1274 & -0.0363 & 0.0198 & -0.1513 \\
0.0291 & -0.4213 & 0.0006 & 0.2248 & 0.0290 & -0.2097 & -0.1079 & -0.0130 & 0.0297 & 0.1502 \\
-0.4423 & 0.1071 & 0.1809 & 0.0553 & 0.0669 & -0.0618 & -0.0906 & -0.0418 & -0.2228 & -0.0472 \\
-0.4254 & -0.1184 & -0.1787 & -0.0092 & -0.0829 & 0.0200 & 0.1217 & -0.2197 & -0.0559 & 0.0630 \\
0.4495 & -0.0763 & 0.0574 & 0.0273 & -0.1861 & -0.0400 & 0.1239 & 0.2109 & 0.0827 & 0.0464 \\
0.3889 & 0.1015 & -0.0614 & 0.0085 & 0.1739 & 0.0012 & -0.1277 & 0.0386 & 0.1913 & -0.0634
\end{array}\right] \\
& A_{d}=\left[\begin{array}{ccccc}
0.3333 & 0 & 0 & 0 & 0 \\
0 & 0.3333 & 0 & 0 & 0 \\
0 & 0 & 0.6000 & 0 & 0 \\
0 & 0 & 0 & 0.6000 & 0 \\
0 & 0 & 0 & 0 & 0.6000
\end{array}\right] \quad B_{d}=\left[\begin{array}{cccccc}
-0.0206 & 0.0206 & 0 & 0 & 0 & 0 \\
0 & 0 & -0.0098 & -0.0098 & 0.0098 & 0.0098 \\
0 & 0 & 0.0003 & -0.0003 & -0.0003 & 0.0003 \\
0 & 0 & -0.0003 & 0.0003 & -0.0003 & 0.0003 \\
0.0004 & 0.0004 & 0 & 0 & 0 & 0
\end{array}\right] \\
& C_{d}=1.0 e+03^{*}\left[\begin{array}{rrrrr}
0.0796 & 0.0000 & 7.3872 & 0.0000 & -5.5493 \\
0.1032 & 0.0716 & -5.9772 & 4.3222 & -1.7160 \\
0.0886 & 0.0442 & 2.2836 & -6.9917 & 4.4907 \\
0.0886 & -0.0442 & 2.2836 & 6.9917 & 4.4907 \\
0.1032 & -0.0716 & -5.9772 & -4.3222 & -1.7160
\end{array}\right] \\
& D_{d}=\left[\begin{array}{rrrrrr}
10.8171 & 3.9903 & -7.0133 & 7.0133 & 7.0133 & -7.0133 \\
6.7151 & -2.1362 & 11.2687 & -8.3349 & -3.0144 & 0.0807 \\
-2.1923 & -9.7904 & -7.9381 & 9.7505 & -5.4144 & 3.6020 \\
-2.1923 & -9.7904 & 3.6020 & -5.4144 & 9.7505 & -7.9381 \\
6.7151 & -2.1362 & 0.0807 & -3.0144 & -8.3349 & 11.2687
\end{array}\right]
\end{aligned}
$$

Acknowledgments

This work was supported by NASA Langley Research Center (NAG1-1056). Experimental data were provided by David E. Cox. The support is gratefully acknowledged.

\section{References}

'Juang, J.-N., Applied System Identification, PTR Prentice-Hall, Englewood Cliffs, NJ, 1994, pp. 229-252.

${ }^{2}$ Huang, J.-K., Hsiao, M.-H., and Cox, D. E., "Indirect Identification of Linear Stochastic Systems with Known Feedback Dynamics," Journal of Guidance. Control, and Dynamics, Vol. 19, No. 4, pp. 836-841. 